\title{
Experiments in sideband suppression on the Los Alamos National Laboratory free-electron laser
}

\author{
Cheryl J. White, Matthew R. Coyle, and Alan H. Paxton \\ Mission Research Corporation, Laser and Optical R\&D Group, 1720 Randolph Road, SE \\ Albuquerque, New Mexico 87105-4245 \\ Patrick G. O'Shea, Stephen C. Bender, Donald A. Byrd, Donald W. Feldman, \\ John C. Goldstein, Eric J. Pitcher, and Thomas J. Zaugg \\ Los Alamos National Laboratory \\ APEX FEL TEAM \\ Los Alamos, New Mexico 87545
}

\begin{abstract}
Two versions of the "Phase-Step Mirror" (PSM), a novel optical component that prevents the formation of sidebands in a Free-Electron Laser (FEL) were lested on the Los Alamos National Laboratory (LANL) APEX FEL. Sideband suppression and frequency control with high extraction efficiency and single line, transform limited operation were demonstrated. The results of our LANL experiments and computer simulations showed that for very high gain applications, the first-order sideband is completely suppressed, but the laser ain is so strong that on about pass 300 the sideband at the second-order or next free spectral range of the PSM appears. This second-order sideband may be suppressed by designing a PSM with grooves having two alternating depths, one chosen to suppress the first-order sideband, and the other, the second-order sideband.
\end{abstract}

\section{THE PHASE-S'TEP MIRROR (PSM)}

Our method for sideband suppression is based on the existence of a low-pass spatial frequency filter in the resonator. The optical beam must have a small diameter in the wiggler region; its diameter is typically larger by at least a factcr of ten in some other section of the resonator. Focusing the beam and propagating it through the wiggler acts as a spatial filter. Increasing the spatial frequency content of the sideband light above the cutoff frequency of the spatial filter while maintaining nearly diffraction-limited performance at the central frequency discriminates against the sideband radiation due to its higher losses at the spatial filter.

Figure 1 is a representation of the spherical PSM. The PSM can be configured ir numerous ways. We have fabricated and experimented with single- and multiple-period versions of both spherical and flat-segmented PSMs. Both versions of the spherical mirror were tested on the LANI, TEI. 
The height of the step is selected to shift the phase fronts of light at the center frequency by an integral number of wavelengths, and to cause significant phaso shifts at the sideband frequency. Thus the sideband light is severely aberrated and cannot be focused into the wiggler while light at the center frequency is uraffected. The height of the raised areas is an integral number of half wavelengths at the center frequency.

The resonator shown in Figure 2 is an almost concentric, asymmetric, standing-wavio, stable resonator, such as that of the LANL oscillator. Suppose that the center wavelength is $\lambda_{c}$ and the sidebands are at $\lambda_{s}=\lambda_{c} \pm \Delta \lambda$. Now suppose that Mirror 1 of Figure 2 is replaced by what we refer to as the "phase-step mirror" shown in Figure 1. It has the same curvature, but (for normal incidence) it has periodic steps of height

$$
\Delta z=n \lambda_{c} / 2
$$

where $n$ is an integer for which

$$
n=\alpha \lambda_{s} / \Delta \lambda
$$

and $\alpha$ is a constant to be chosen. $\alpha=0.5$ creates an intensity null at the focus in the wiggler for the sideband frequency. Nominal values for the LANL, oscillator are $\lambda_{r}=3.0 \mu \mathrm{m}$ and $\Delta \lambda=0.0285 \lambda_{c}$. Using these values and choosing $\alpha=0.5$ gives $n=18$ and $\Delta z=26.10 \mu \mathrm{m}$. Reflection of a spherical wave of wavelength $\lambda_{r}$ from the PSM causes no aberration because the difference in path length of light reflecting from any of the step: is an integral number of waveiengths. We have assuned that the step height is much less than the pulse length of tie FEL so that effects due to the finite pulse length may be ignored. Reflection of a spherical wave of wavelength $\lambda_{0}$ from the mirror aberrates it severely, introducing a phase shiet of $2 \pi \alpha$ for every half period of the mirror step pattern. This functions as a phase grating with miltwwelength groove depth and a very low period, but affords far fewer sites for possible damage than typical low period metallic gratings. The mirror steps need not follow straight lines. This configuration allows simple analysit, but the mirror fabricated for the LANI, FEL experiments has a concentrir, circular groove pattern over the central centimeter of the mirror.

\section{THE LANL AIPX FEI}

The Los, Alamos APEX lifil, was created as an advanced technology test-bed for the Bowing

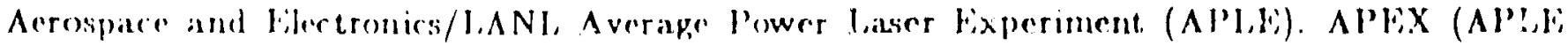
f'rototype lixperiment) is a photeinjector-driven Flil, that is carrently operated as an oscillator with

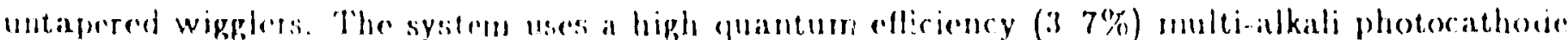

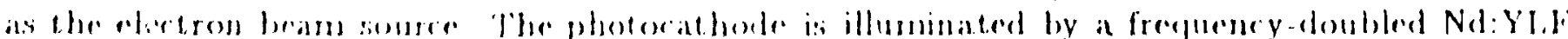

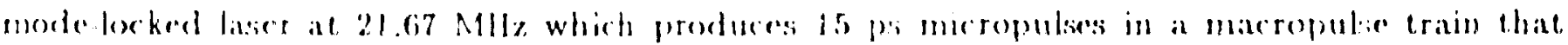

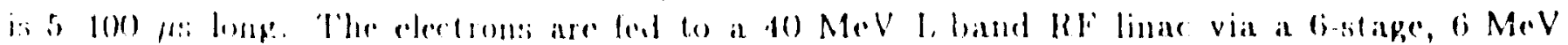
photominertor invanted at los: Alamesi 
A uniform $\mathrm{SmCo}_{5}$ Halbach undulator was used. It was $1.00 \mathrm{~m}$ in length with 49 periods, each $2.05 \mathrm{~cm}$ in length. The gap was $4.2 \mathrm{~mm}$ and the on-axis magnetic field was $0.64 \mathrm{~T}$, resilting in a vector potential $a_{w}$ (peak) $=1.2$.

Our experiments observed the effects on the optica! output energy, spectral content, spatial intensity distribution, and temporal intensity evolution when we replaced the cavity's usual multilayer dielectric (MLD) high (back) reflector with two versions of our PSM. The electron beam energy and macropulse length were varied and the e-beam extraction efficiency measured. The small signal gain and cavity losses were measured.

\section{THE APEX OSCILLATOR}

Figure 2 is a schematic of the LANL APEX resonator. It is an asymmetric, almost concentric, standing-wave, stable resonator. The optical waist occurs at the wiggler center, which is $0.5 \mathrm{~m}$ upstream from the geometric center of the $6.918 \mathrm{~m}$ resonator. In the nominal configuration, the

resonator consists of an output coupler and a high reflector with $4.0 \mathrm{~m}$ and $3.03 \mathrm{~m}$ concave spherical radii of curvature, respectively. Hoth are made on calcium fluoride $\left(\mathrm{CaF}_{2}\right)$ substrates and coated with a MLD for $R=99.6 \%$ reflectivity and $T=0.4 \%$ transmissivity at $3 \mu \mathrm{m}$. Gaussian bean spot sizes (radii) at the mirrors are $4.44 \mathrm{~mm}$ and $5.89 \mathrm{~mm}$. The resonator mirrors have suffered severe coating and substrate damage when the macropulse-average intracavity mirror-fluences exceed $750 \mathrm{~kW} / \mathrm{cm}^{2}$. $^{2}$

Our sideband suppression technique is based on the existence of a low-pass spatial frequency filter in the resonator. In the $l \Lambda N$ N device, the wiggler aperture serves this function. The optical beam is focused to create a small diameter in the wiggler region to provide adequate overlap with the electron beam. A FSM will suppress the sidebands in any FEl, resonator if it can be included in a region where the optical beam size is much larger than its size in the wiggler. 'The beam waist, $w$, in this resonator is about $0.6 \mathrm{~mm}$. The aperture of the $1 \mathrm{~m}$ wiggler is about $5 u$.

\section{LANL FEI, BASELINE PERFORMANCE}

The data in Figure 3(a), (b), (c), and (d) establish the basclime performance of the I,ANI, FFi, without the PSM. There is close agrement between the calculated and observed baseline specerum of the oscillator with standard optics de:scribed above. Figure $3\left(\right.$ a) depicts the resilts of an f fil, ${ }^{3}$ simulation of the buili-up of intracavity power as a function of pass number (time). At about 16 psece, the sideband onsirt is observed as a sharp rise in the optic al power, which penks at nearly 1.5 (iW some 30 pser into the macrosulse. figure $3(b)$ shows the corresponding experimental measurement of intracavity power; it showe strong sideband onset at about 15 pesec and a peak intracavity power of 1.5 ( $: W$. Fipure $3(c)$ showa a Flill' simulation of the optical spectrum and shows a strong, sideband frature at $3.5 \%$. Figure $3(d)$ shows the corresponding experimental optic al output spectrum. The spectrum is hroad and chaotic, with a strong sideband feature at $3.5 \%$ for a center wavelenpth of $2.90 \mu \mathrm{m}$. This experimental datas wat obtained with the aarne diapnextic:a used in our experiment.: with the l'sim:i. 


\section{EXPERIMEN'TS WITH THE MULTIPLE-PERIOD SPHERICAL PSM}

The first series of experiments was conducted using a multiple-period, concentrically grooved, spherical PSM. It has the same $4.0 \mathrm{~m}$ radius of curvature as the standard MLD optic. This allowed direct replacement of the standard optic with the PSM. The MLD mirror that previously served as the output coupler was replaced, and the output through the mirror with the $3.03 \mathrm{~m}$ radius of curvature was routed to the optical diagnostic equipment. A $26.10 \pm 0.0750 \mu \mathrm{m}$ step height was our design goal obtained from the LANL oscillator specifications and the spectral data shown in Figure 3.

The mirror was diamond-turned on a copper substrate and coated with bare gold for maximum reflectivity at $3 \mu \mathrm{m}$. Its reflectivity was measured to be $>97 \%$ over a wavelength range of several hundred nanometers. A special diamond tool was designed and fabricated, and a modifed plunge-cutting technique was devised to fabricate the PSM for this project.

While the individual groove profiles and figure were very good, with RMS surface roughness $<\lambda / 100$, through most of the development we did not have access to an adequate diagnostic to measure the variation of depth from groove to groove. It was assumed because the tool that cuts the grooves is interferometrically driven that while the actual groove depth we obtained might vary within the allowed tolerance, that the variation would be systematic and therefore the groove depin would be uniform from groove to groove. Last-minute access to a contact profilonetcr with an electrostatic probe showed this to be untrue: the groove depths varied from 31.8359 to $33.7785 \mu \mathrm{m}$, resulting in an average depth of $32.5455 \mu \mathrm{m}$, with a standard deviation of $0.5287 \mu \mathrm{m}$. Scheduling constraints on the LANI. FEL prohibited another fabrication run, but it should be possible to use such data to adjust the tooling program to produce a mirror with acceptable uniformity of depth groove to groove.

Because of the nonuniformity of the grooves, the cavity losses were quite high, on the order of 60\%, as measured by the cavity ring-down. John Goldstein of LANL, ran one-dimensional nurnerical simulations, modeling the filter function of the PSM as $\cos ^{2}(\pi \alpha)$. He found that for such high loss, the laser would not support sidebands, with or without the filter function. Thus, this data alone does not allow us to stite conclusively that the mirser demonstrated sideband suppression. However, narrow, single line, performance with high electron beam extraction efficiency and strong frequency selectivity was ohserved.

Tuning, behavior consistent witis that expected from an etalon-like devire was observed. That i: as the r-beam energy was varied, wavelength tuning over a small range then a frequency jump to the next passhand or free sipectral rane resulted. This indicates lasing was controlled by the ISSM.

The trates: in Fipures 4 and 5 record the ontput of a meroury-doped permanium detector which measures the intracavity power. Figure 4 records the build up of intracavity power at the suart of the macropulse. A logarithmic fit of this "ring-up" pivess a measure of the single pass, 
smail-signal gain, which was found to be $\sim 150 \%$. At the end of the macropulse, when the e-beam is "turned off," the rate of decay of intracavity power is called the "ring-down," shown in Figure 5. A logarithmic fit of the ring-down gives the total cavity loss, which was measured at $\sim 65 \%$. For these and the following spectra, the electron beam energy was $38.6 \mathrm{MeV}$; the macropulse length was $30 \mu \mathrm{sec}$; and the micropulse charge was $2 \mathrm{nC}$.

The trace in Figure 6 is the energy distribution function of the electron-beam in a non-lasing condition. Energy increases to the right. To obtain the distribution, a shutter is inserted in the resonator, preventing lasing. The e-beam is passed through a bending magnet and into a multichannel analyzer, creating the spectrum observed as the spot in the middle of the picture. The curve is a digitized profile of that energy distribution.

The trace in Figure 7 shows the shift in distribution of the energy of the electrons under lasing conditions when the shutter is opened. The shift in the centroids of these two traces gives the extraction efficiency. As part of the electron population gives up energy to light, two distinct distributions are observed. The extraction efficiency for this shot was measured at $0.98 \%$. Values as high as $1.02 \%$ were recorded. These values are as high as any ever observed on this device.

Figure 8 shows the wavelength dependence of the optical output as the electron beam energy was tuned from $37.7 \mathrm{MeV}(3.096 \mu \mathrm{m})$ to $39.9 \mathrm{MeV}(2.804 \mu \mathrm{m})$. The behavior of the output wavelength demonstrates strong frequency selectivity due to the PSM. We measured two shifts of $\sim 5.234 \%$, which correspond to a jurnp of $0.193 \mu \mathrm{m}$. This agrees well with theory, which predicts successive passbands separated by $\sim 0.2 \mu \mathrm{m}$ for an ideal grooved mirror with our design specifications. This becomes spread over a calculated range of about 0.15 to $0.22 \mu \mathrm{m}$, due to the nonuniformity of the grooves. The filter function of the PSM can be modeled to first-order as $\cos ^{2}(\pi \alpha)$, represented by the dotted line. For this data, the dutted line represents successive free spectral range passbands at intervals of $0.185 \mu \mathrm{m}$, the average of the range from 0.15 to $0.22 \mu \mathrm{m}$. As the e-beam energy is tuned, the output wavelength remains close to the minimum loss point (maximum transmission through the wiggler focus). It then jumps across the region of maximum loss (transmissicn minimum) and once again remains near the next minimum loss value. This is consistent with the PSM forcing lasing within a single passband. When lasing in the adjacent passband can no longer be supported, the laser frequency-hops to the next free spectral range.

The beam encrgy was fixed at $39.7 \mathrm{MeV}$. Energy extraction efliciency was measured as the macropulse wilth was varied from 5 to $40 / \mathrm{ss}$. As expected, a monotonically increasing dependence was cheserved. This data is plotted in Figure 9(a). The macropulse width was then fixed at 30 pl:;, and the beam energy was varied from $~ 30.5$ to $41 \mathrm{MeV}$ (Figure $9(b)$ ). Again, the filter function of the PSM is suggesied by the sketehed chirve.

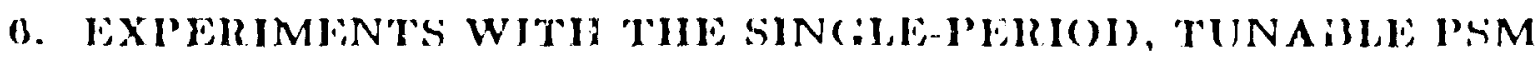

The next series of experiments were conductert using a sinple period, tunable spherical 'sM

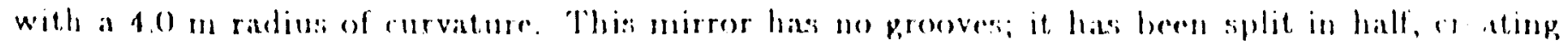


a single phase-step. ${ }^{41}$ One segment can be pistoned relative to the other segment, which allows any desired step height to be obtained. This mirror has the advantage of ease of fabrication and broad, continuous tunability. Complete sideband suppression and transform limited, single-line, wavelength tunable performance were demonstrated for moderate small-signal gain (up to 100\%). The disadvantage of a single-period, however, is that it cannot provide sufficient contrast to assure complete sideband suppression when operated with extremely high small-signal gain.

The split mirror was first adjusted for zero step height. This allowed us (unlike the case of the grooved mirror) to observe the baseline behavior of the laser without the PSM immediately prior to introduction of a step-height of $26.10 \mu \mathrm{m}$. Figure $10(\mathrm{a})$ is the build-up of intracavity power, or "ring-up", and shows a small-signal-gain of $35 \%$. The total cavity loss for the split mirror ranged from $\sim 5$ to $7 \%$. This range is due to small variations in alignment over the series of experiments. Figure $10(\mathrm{~b})$ shows a total cavity loss of $5.8 \%$. For these and the following spectra, the electron bearn energy was $39.4 \mathrm{MeV}$, the macropulse length was $20 \mu \mathrm{s}$, and the micropulse charge was $2 \mathrm{nC}$.

The electron-beam energy extraction efficiency was measured for these conditions. Figure 11(a) is the electron energy distribution in a "no lasing" condition, while the lower trace in Figure 11(b) shows the redistribution of electron energies with lasing. Calculation of the centroid shifts in these electron energy distribution spectra gives an e-beam extraction efficiency of $\sim 0.66 \%$ for this low small-signal gair. case.

Figure 12 shows the baseline optical output spectrum. For this high gain, zero step height condition, sidebands are present; the spectium is broad and chaotic.

A step height of $26.10 \mu \mathrm{m}$ was introduced. The sidebands were entirely suppressed. A smallsig: $=1$ gain of $70 \%$ and a ring-down loss of $6.3 \%$ were measured. Fnergy extraction efficiency was $\sim 1 \%$. A single-line, transform limited optical spectrum was observed at a fundamental wavelength of $2.9 \mu \mathrm{m}$. The step height was then varied. Significant line width narrowing, tunability of the output wavelength and complete suppression of the sidebands was demonstrated. Figure 13 shows several transform limited shots taken as the step-height of the PSM was tuned.

It becane clear after a number of step-height adjustments were made that we were losing alignment. The cavity losses incruised to $\sim 20 \%$ and the extraction efficiency fell to $\sim(0.5 \%$. Correspondingly, the output power chereased and sidebands began to appear. Part of this was due to hysteresis in the piezos which introduced tip-tiit errors as the mirror was pistoned. Another source of alignment problents is thermally induced changes in the cavity. At one point lasing, was lost and it was found that the cavity had 'Grown' by more than $100 \mu \mathrm{m}$. 'Tip-tilt : djustments were also reculued. The cavity can easily be pue under active control as well as the controls which definition and maintain mir ror step height.

We helieve mest of the operationsl difficulties and irreproducibility experienced arise from effects which could be eliminated by placing the system under active computer control. 'These 
effects are several: Some are variations in cavity length, e-beam energy, alignment of the cavity, and alignment of the e-beam through the wiggler.

The final series of experiments were conducted by setting the split mirror to a step height of $26.10 \mu \mathrm{m}$ prior to pump down. When the FEL was brought up, lasing was obtained immediately. Minor tip-tilt adjustments were made to optimize the optical output. The fundamental came up in a single-line, with narrow bandwidth at $2.794 \mu \mathrm{m}$ for an e-beam energy of $39.9 \mathrm{MeV}$. This agrees with the data obtained from the grooved mirror $\{\lambda(E)\}$ (see Figure 8 ) for this set of operating conditions.

The cavity length was increased by $20 \mu \mathrm{m}$, producing a slightly broader spectrum. The center wavelength shifted to $2.804 \mu \mathrm{m}$. Stiil no sidebands were observed. The e-beam emittance and alignment were improved, which increased the small-signal gain to $\sim 100 \%$. While the spectrum was much more highly ordered than that without the PSM (see Figure 12), sidebands at about. $6 \%$ of the fundarnental, the next free-spectral range (second-order), did appear.

Figure 14 shows a series of shots where the step height was fixed at $26.1 \mu \mathrm{m}$ and the energy of the electron bearn was varied from $39.9 \mathrm{MeV}$ to $39.4 \mathrm{MeV}$. The effect on the output optical spectrum was observed. For a fixed step height, within a single free spectral range, we expect onl; one small frequency range where efficient sideband suppression occurs. The behavior observed in this series of shots shows increasing suppression efficiency as adjustment of the e-beam energy and cavity length brings the laser closer and closer to this ideal operation point.

Throughout this series of shots we had difficulty in maintaining a consistent match between the electron bearn and light field. A snowstorm and attendant snow plows served to amplify the errors introduced by the factors discussed above and essentially every knob in the facility was tweaked diring the course of the experiment. For this reason, we only report a general trend in the evolution of the data we observed.

Onr alculations show that for a fundamental at $2.9 \mu \mathrm{m}$ and sidebands at $2.85 \%$, a step height of $26.10 \mu \mathrm{m}$ should provide maximum suppression. For a fixed step height of $20.10 \mu \mathrm{m}$, the spectra in Figure 14 show that as the e-beam is tuned to $39.4 \mathrm{MeV}$, bringing the principal output nearer to $2.9 \mu \mathrm{m}$, the sidebands are more efficiently suppressed and the power in the principil wavelength is increased by a factor of $\cdots 5$.

We ran one-dimensional numerical simulations to model the PSM. $\Lambda$ function of $\cos ^{2}(\pi \alpha)$ was imposed on the usual code to represent the filter action of the PSM. Results were obtained for thece values of total cavity loss, $60 \%, 25 \%$, and $5 \%$ and are in agreement with the experimental results obtained with the grooved mirror $(60 \%)$ and the split mirror $(5 \%)$. 


\section{CONCLUSIONS}

The grooved, multiple-period PSM demonstrated strong frequency selection and therefore control of lasing by the PSM, but failed to provide conclusive proof of sideband suppression due to nonuniformity of groove depths which led to high cavity losses. One-dimensional simulations indicate no sidebands are generated for the resulting cavity intensity, with or without the PSM. However, presence of the PSM resulted in a much narrower spectrum with the spectral intensity almost doubled for the remaining peak. Thus, even for FELs that do not generate sidebands, the PSM can be useful to narrow the spectrum and enhance the interaction with a resonance of importance in chemical, materials, or biological experiments.

The single-period PSM demonstrated complete sideband suppression for moderate small-signal gain. Our experiments also demonstrated that the split mirror's continuously adjustable step height allows tunable, single-line, narrowband operation. Numerical simulations for operation with high small-signal gain indicate that the single-period may not provide sufficient contrast for complete sideband suppression. The single-period PSM data supports the conclusion that a grooved PSM with more uniform groove depths will extend the range of complete sideband suppression to higher small-signal gain.

We believe that due to advances in fabrication technology that resulted from this project it is now possible to fabricate a more uniform grooved mirror with two step-heights to suppress the formation of first- and second-order sidebands. Additionally, it now appears possible to fabricate a tunable, multiple-period PSM.

\section{ACKNOWLEDGEMENTS}

The authors wish to thank Carl Moody of the U.S. Army Strategic Defense Command, Huntsville, AL, for his enthusiasm, support, and assistance; Timothy L. Boyd for contributions to the preliminary development of the techniques used in these experiments; Robert $\mathrm{B}$. Michic for valuable technica! discussions; Teresa $L$. Neudecker and David R. Collins for technical assistance.

This program was sponsored by the Strategic Defense Initiative Organization (SDIO) and managed by the U.S. Army Strategic Defense Command (SDC) under a Phase. II DoI) Small Business Innovation Research (SBIR) project.

\section{REFERENCES}

1. A. H. Paxton and M. J. Schmitt, "Sideband instability in free-electron lasers -a new technique for suppression," HEEF J. Quantum Electron., Vol. 26, pp. 1167-1172, 1990.

2. P. G. O'Shea, S. C. Bender, B. F. Carlsten, J. W. Early, D. W. Feldman, R. B. Feldman, J. C. Goldistein, K. I'. McKenna, R. Martineau, E. J. Pitcher, M. J. Schmitt, W. F. Stcin, M. 1). Wilke, and T. J. Zaugg, "Performance of the APFX free-electron laser at Los Alamos National Laboratory," accepted for publications in Nuclear Instruments and Methols in Physics Research A, 1993. 
3. J. C. Goldstein, B. D. McVey, R. L. Tokar, J. C. Elliott, M. J. Schmitt, B. E. Carlsten, and L. E. Thode, "Simulation codes for modeling free-electron laser oscillators," in Modeling and Simulation of Laser Systems, SPIE Proc. 1045, pp. 28-35, 1989.

4. J. E. Sollid, D. W. Feldman, and R. W. Warren, "Sideband suppression for free-electron lasers, ${ }^{n}$ presented at 1988 Free-Electron Laser Conference, Jerusalem, Israel, 29 August-02 September 1988.

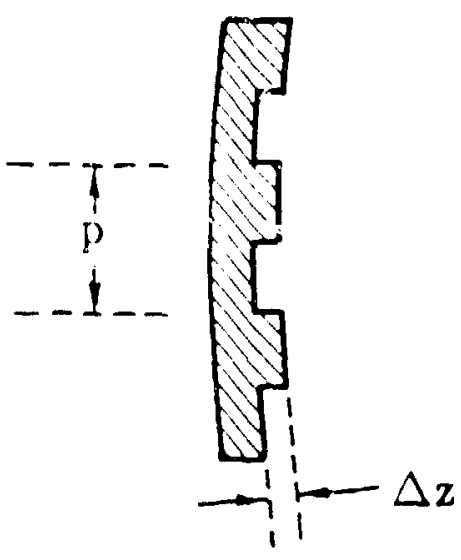

Figure 1. Phase step mirror. The steps are raised a distance $\Delta Z$ above the spherical reference surface. The step height is selected to shift the phase fronts of light at the center frequency by an integra! number of wavelengths and to cause significant phase shifts at the sideband frequency.

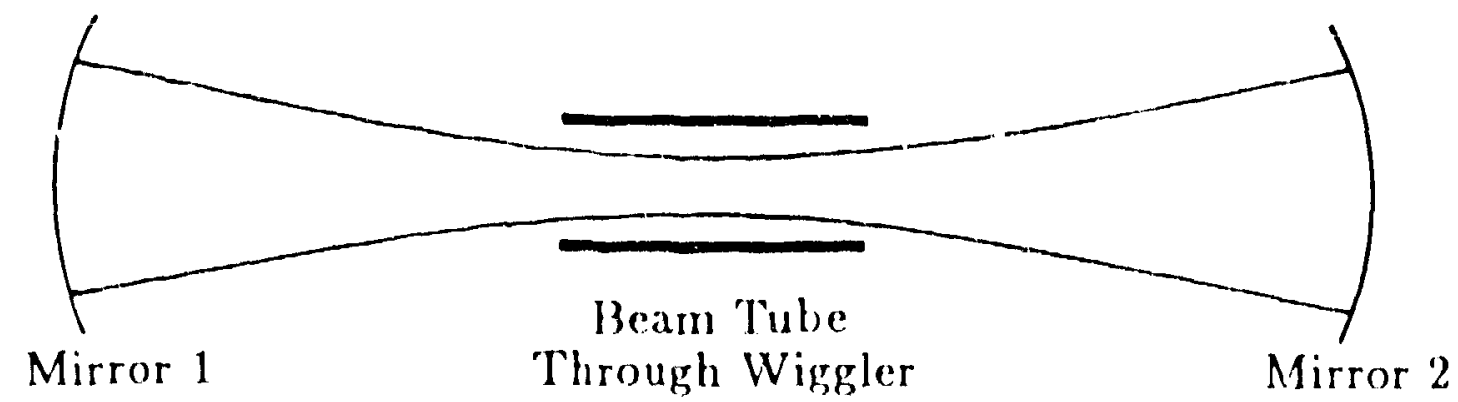

Figure 2. Stable resonator showing beam tube through wiggler in the vicinity of the waist. 


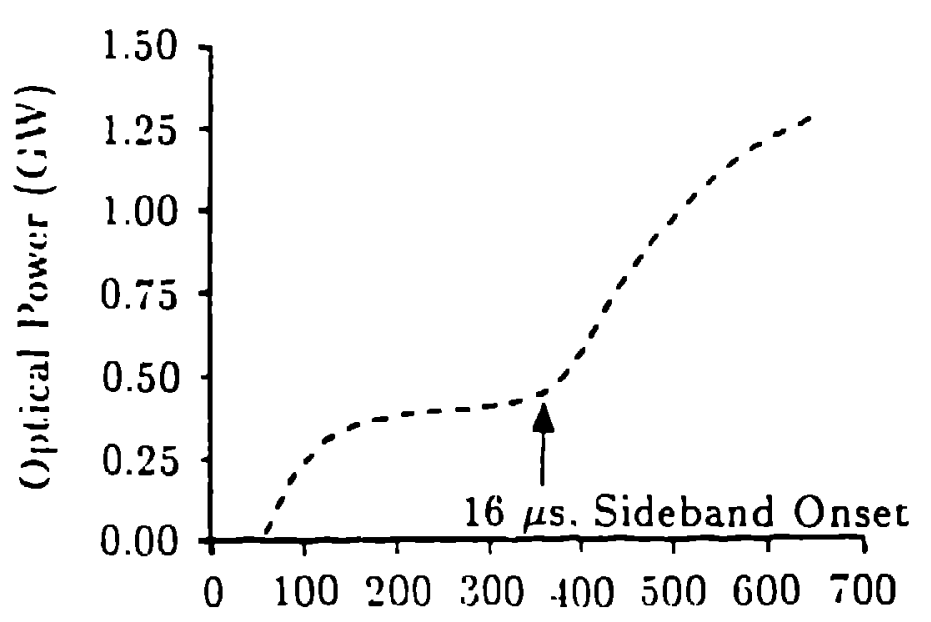

iVirgler Exit Pass Number

(a)

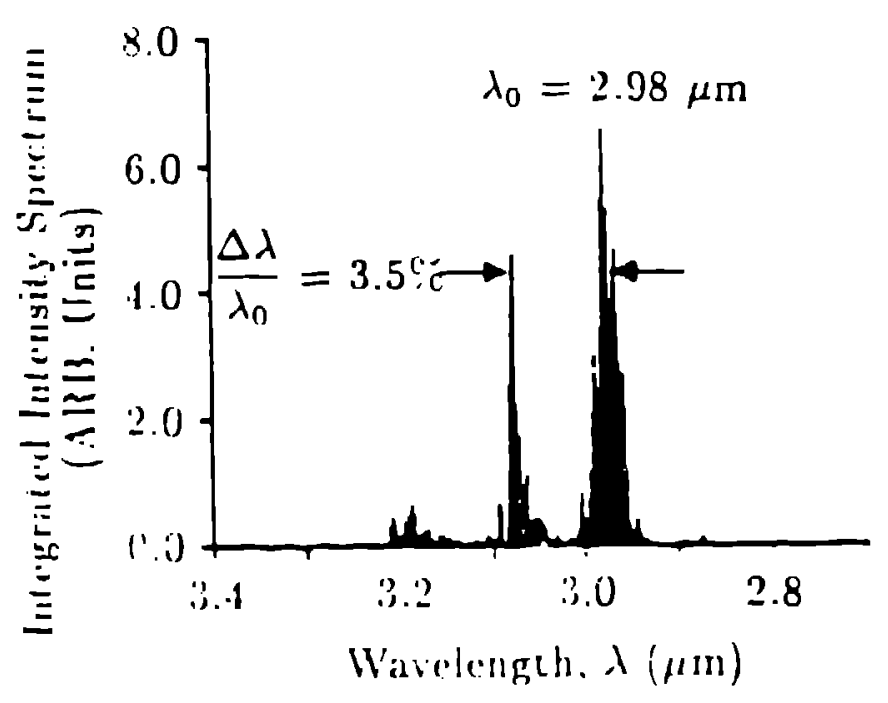

(c)

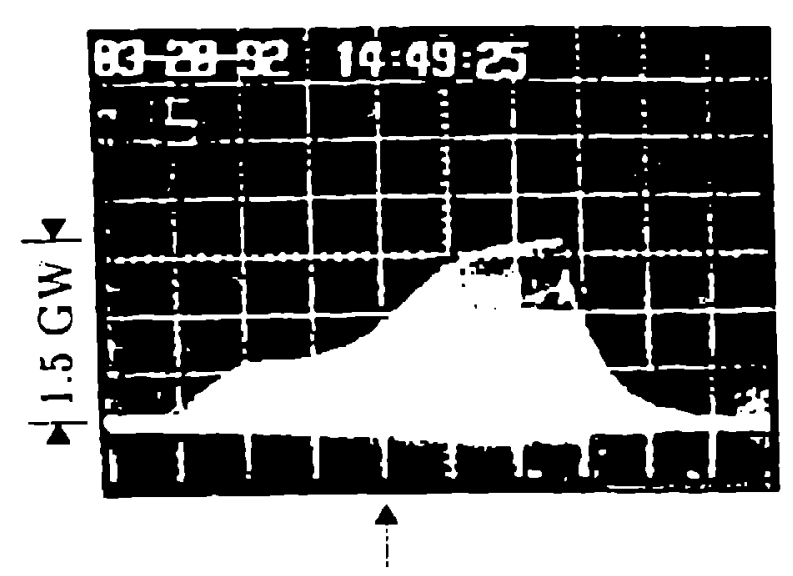

$15 \mu \mathrm{s}$, Sideband Onset

(b)

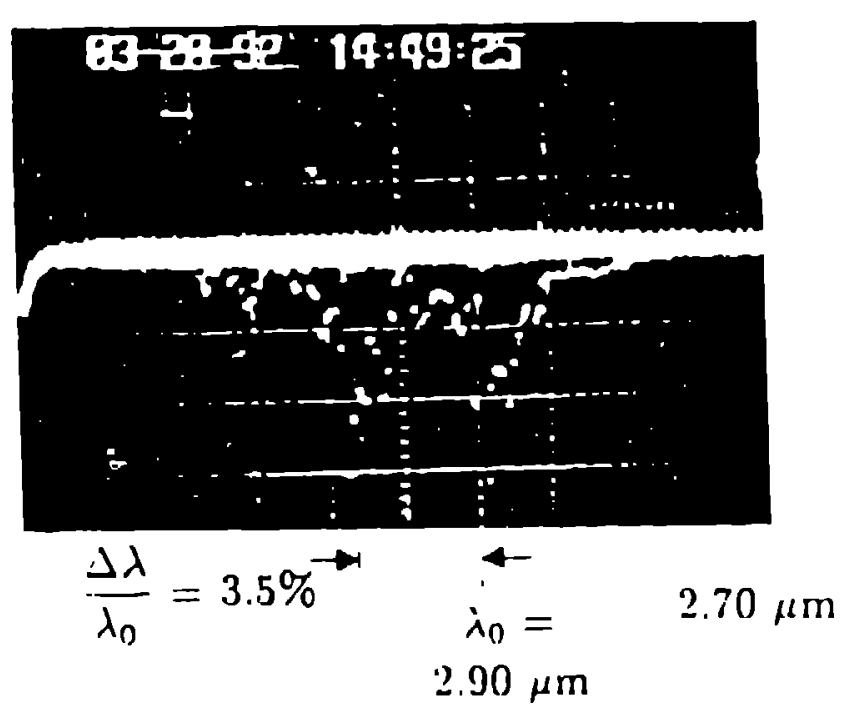

(d)

Figure 3. (it) Optical power. obtained from an FELP numerical simulation. and (b) measured experimentally. (c) Intensity spectrum integrated over the macropulse length, obtained from an FELI' numerical simulation, and (d) measured experimentally. 


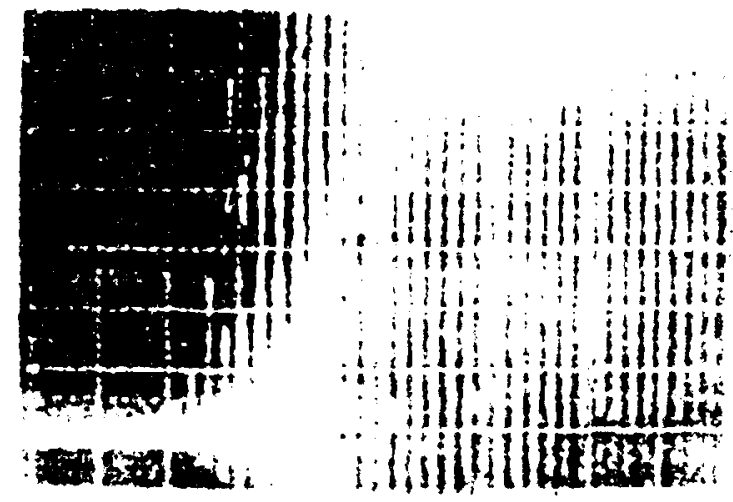

Figure 4. Build-up of intracavity power showing a small signal gain of about $150 \%$.

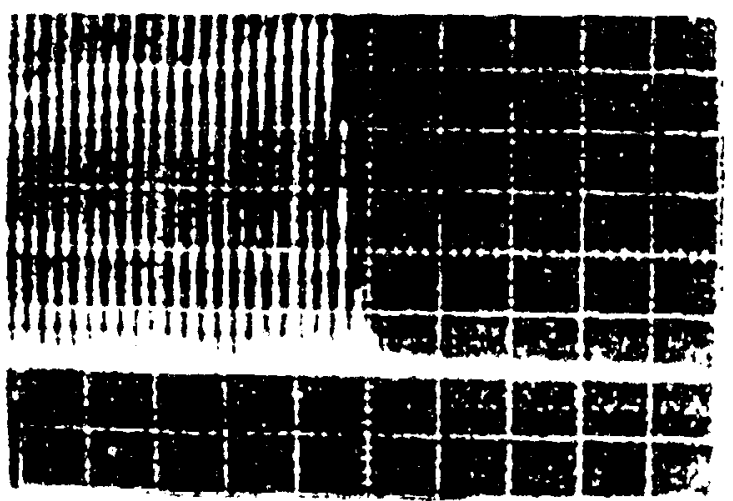

Figure 5. Ring down measurement indicates approxımately a $65 \%$ Loss/Pass.

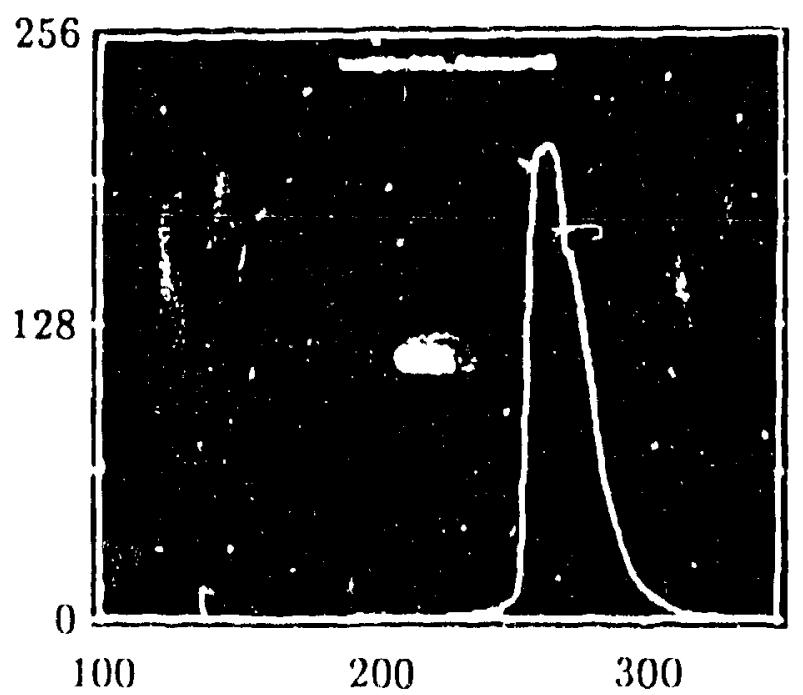

Figure 6. Lilectron beam energy profile--lion lasing condition. 


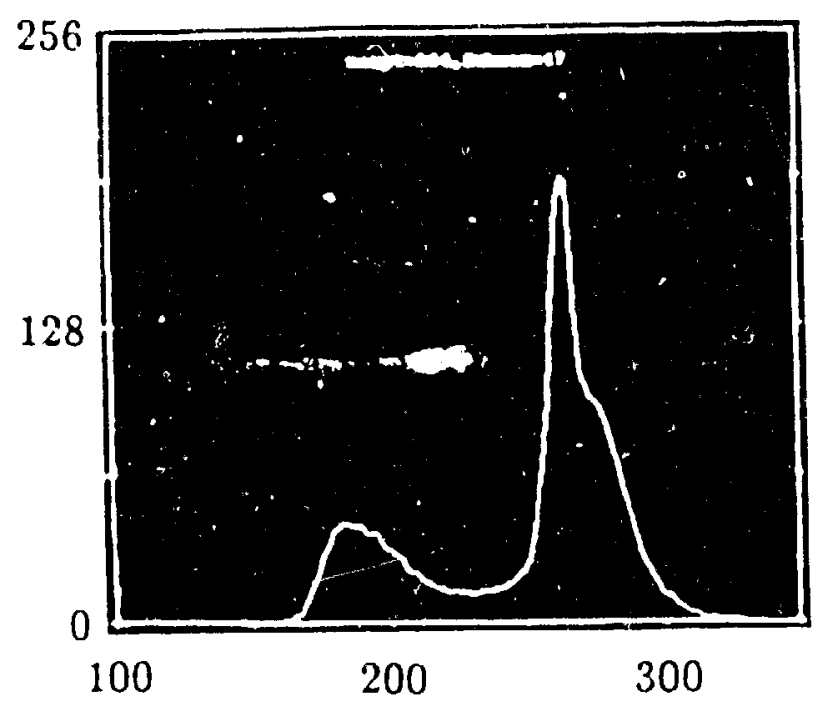

Figure 7. Electron beam energy profile-lasing.

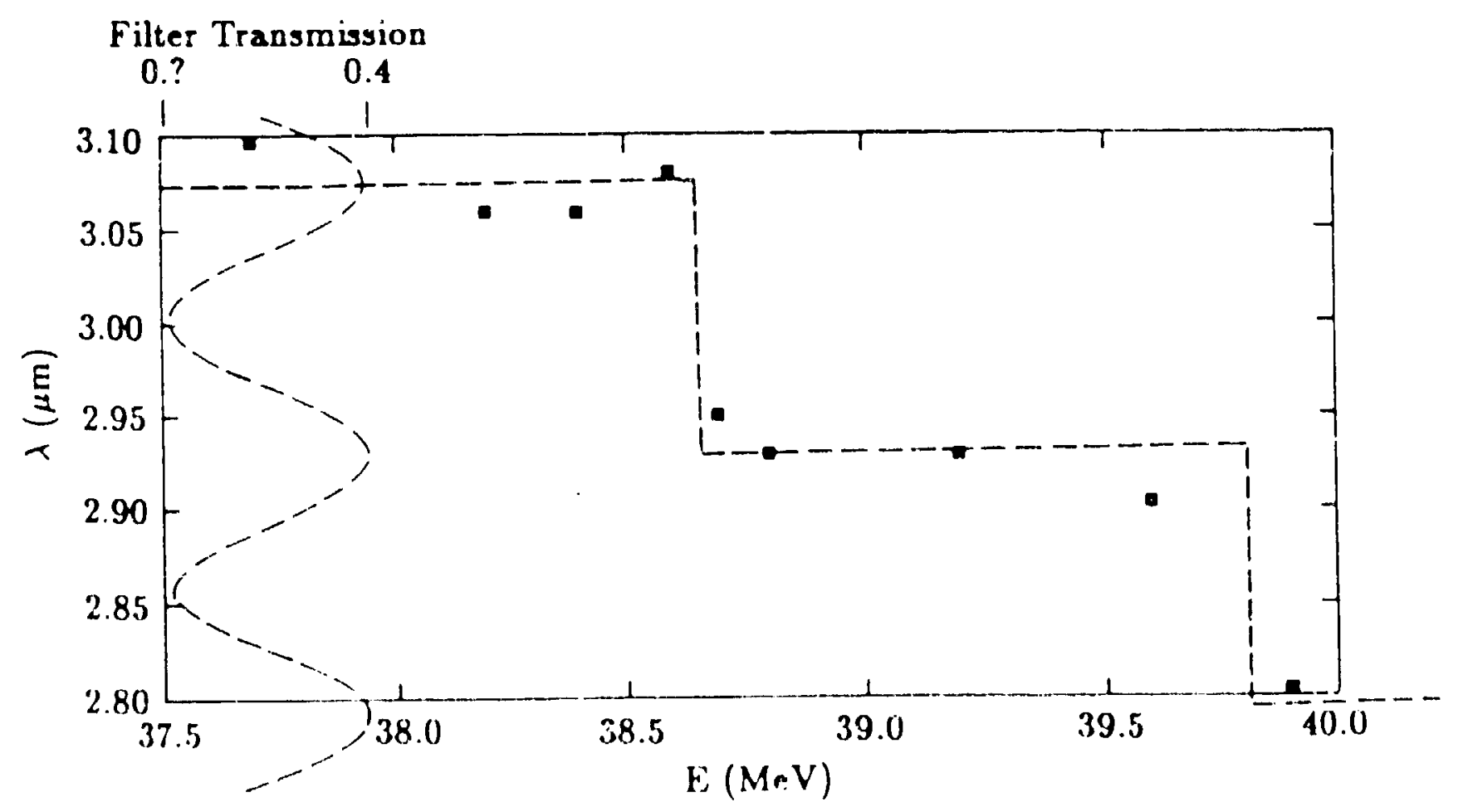

Figure 8 . Wavelength shift as a function of electron beam energy. Filter function is plotted along the vertical axis. Dashed straight lines show the wavelengths of minimum lossi corresponding, to the filter function. 


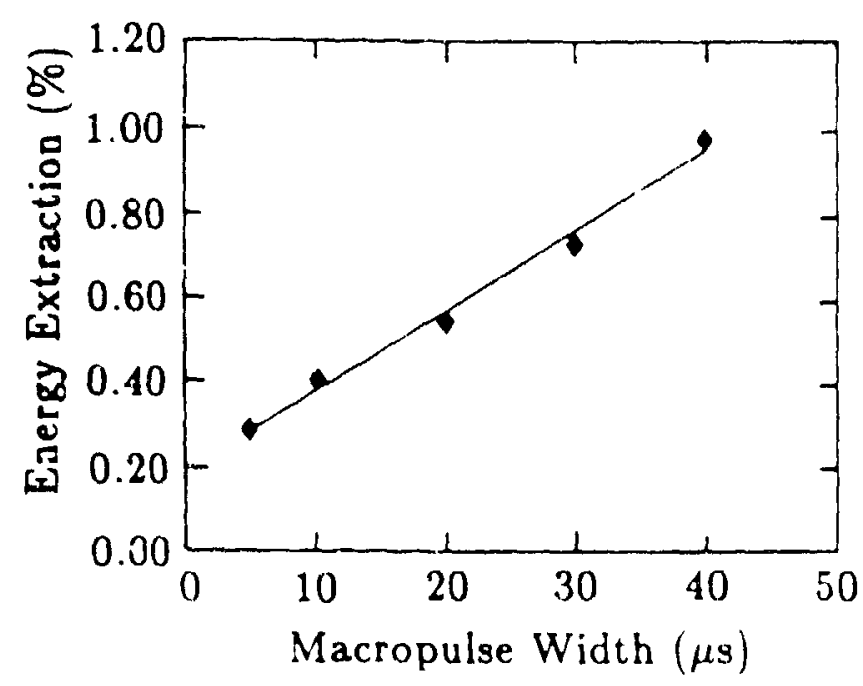

(a)

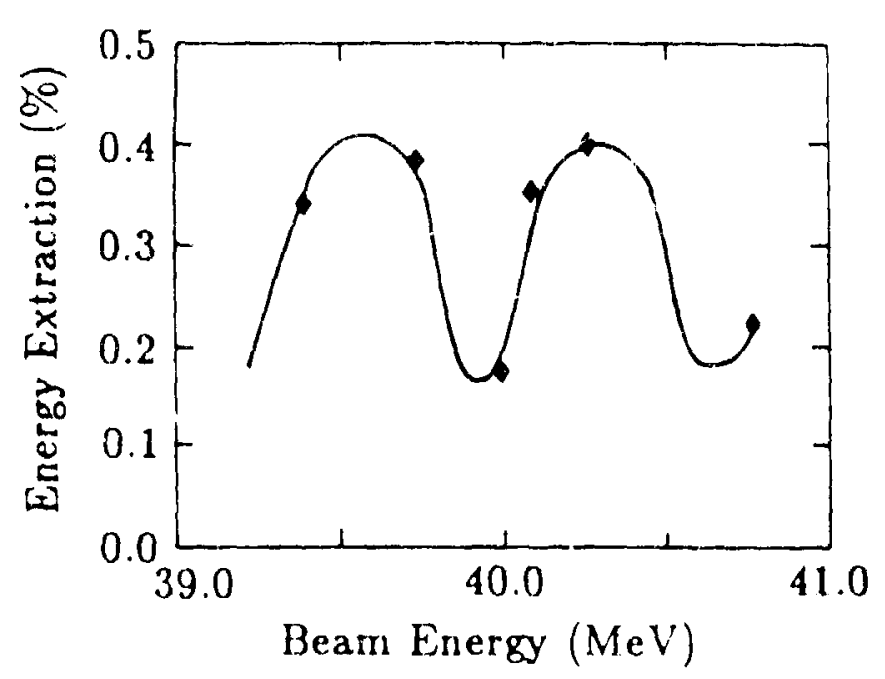

(b)

Figure 9. (a) Energy extraction versus macropulse width. (b) Energy extraction versus beam energy including a sketch of the filter function.

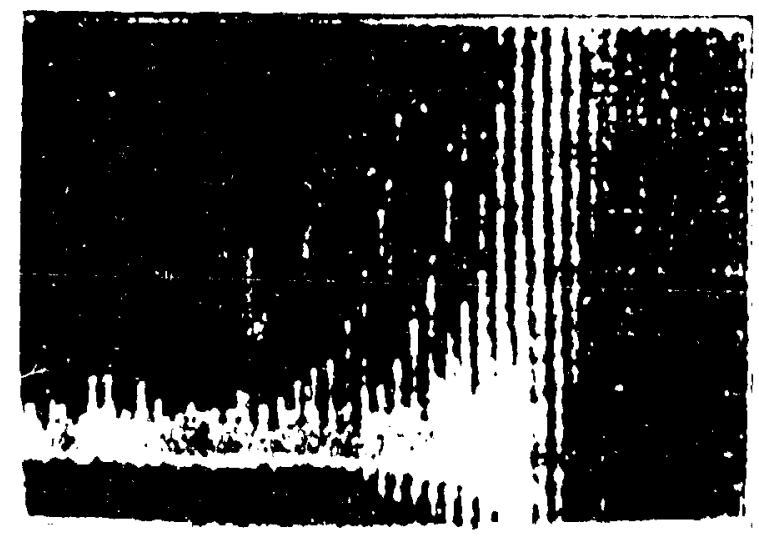

(a)

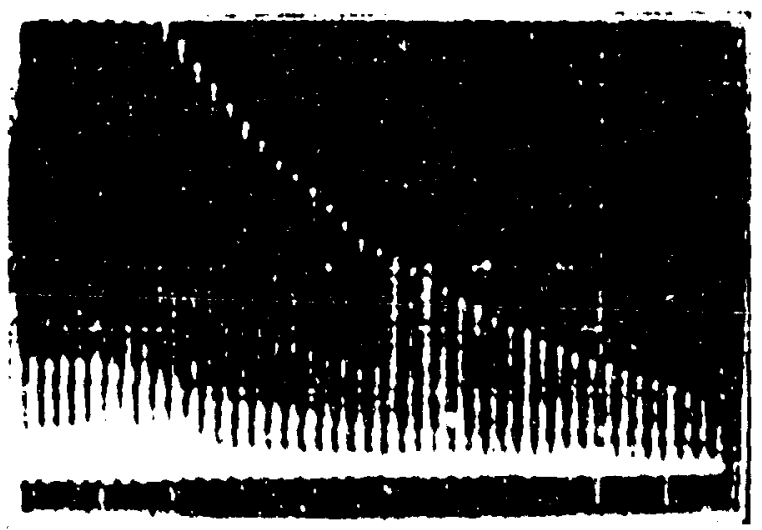

(b)

Fipure 10. (a) Small sipnal pain measurement - $35 \%$ $-5.8 \%$ l,oss/ Yassi.

(b) Ring down measiurement indicate:s 


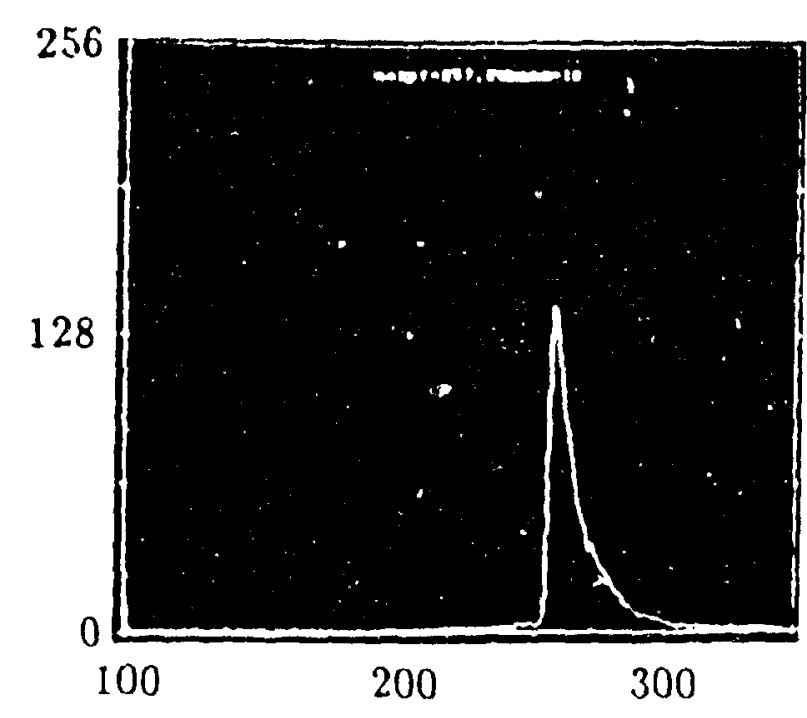

(a)

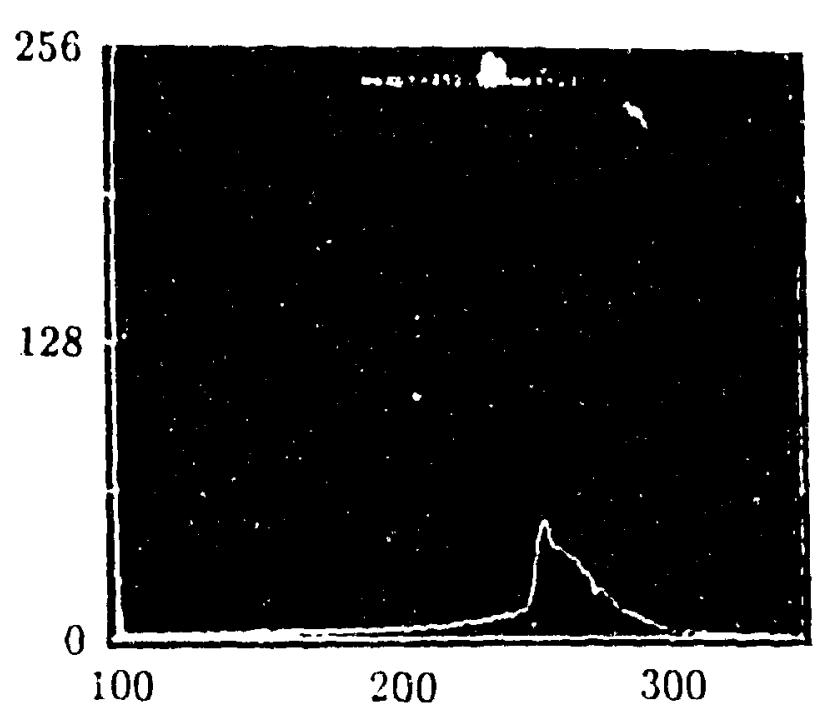

(b)

Figure 11. (a) Flectron beam energy profile-non lasing condition.

(b) Electron beam anergy protile lasing.

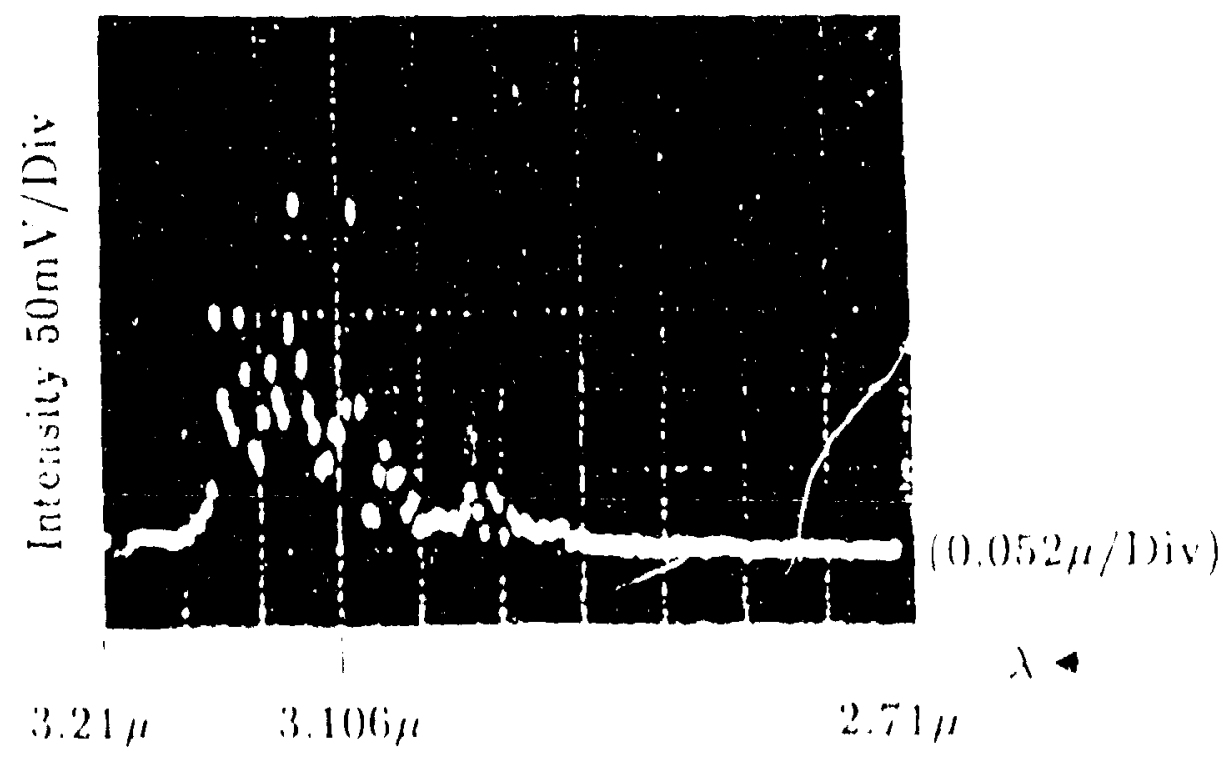

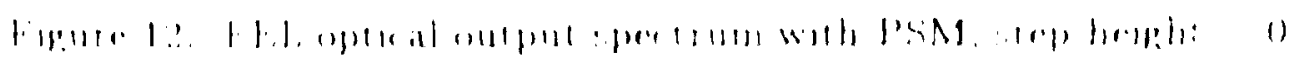




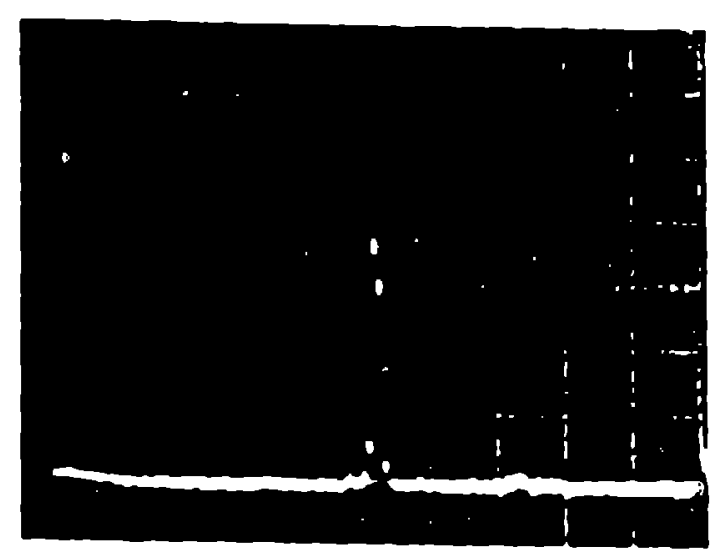

(a)

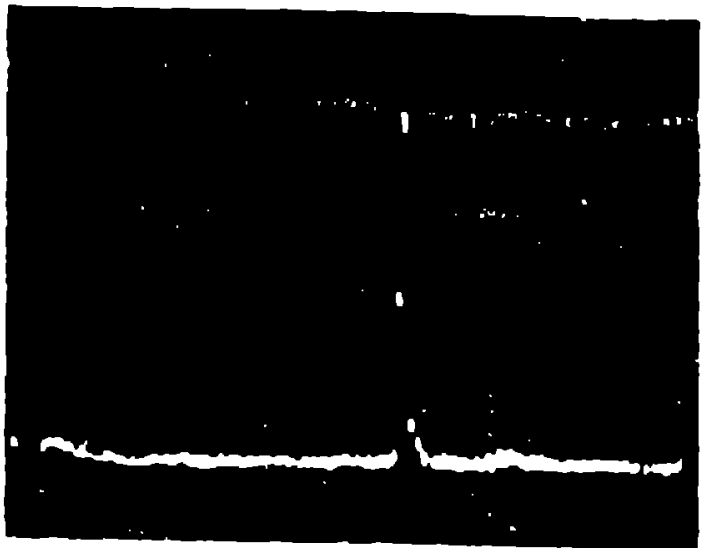

(b)

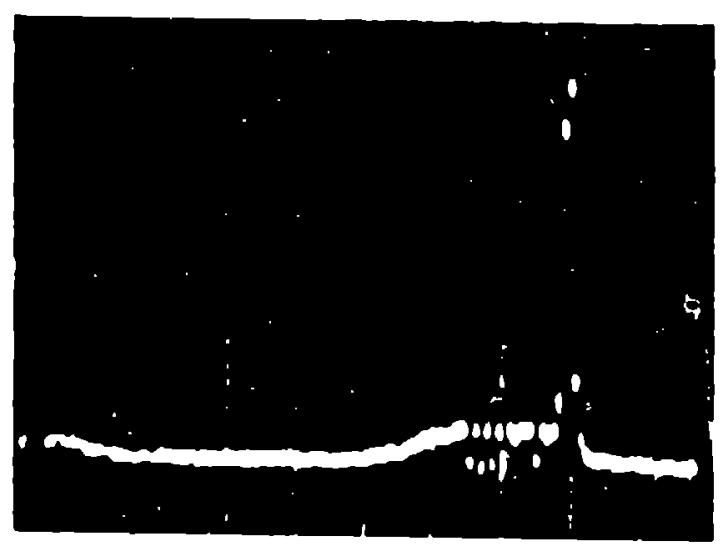

(c)

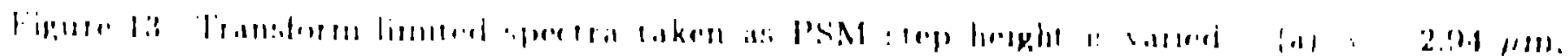
(b) $\lambda$ a.91 mm. (1) A $2.78 \mathrm{~mm}$. 


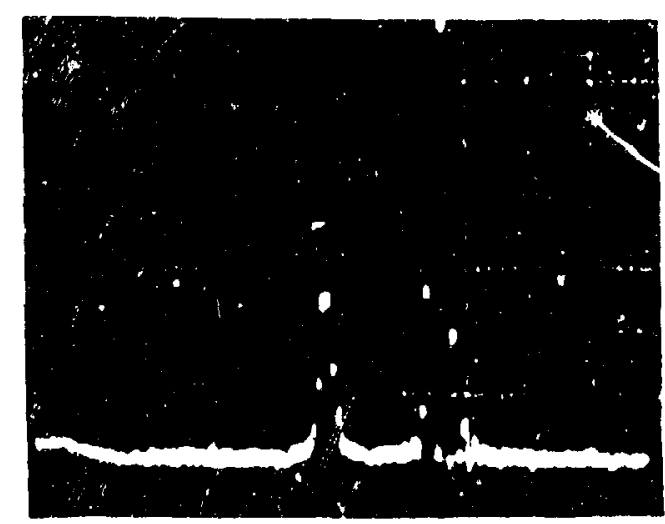

(a)

$$
\begin{aligned}
& E=39.9 \mathrm{MeV}, \\
& \lambda_{1}=2.966 \mu \mathrm{m}, \\
& \lambda_{2}=2.867 \mu \mathrm{m},
\end{aligned}
$$

vertical scale $=10 \mathrm{mV} /$ Div.

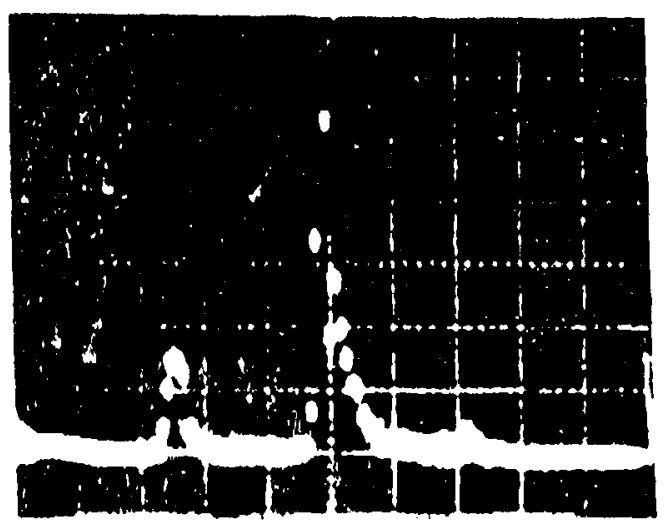

(c)

$$
\begin{array}{ll}
\lambda_{1} & 3.08 \mathrm{~mm}, \\
\lambda_{2} & 2.051 \mathrm{~mm}, \\
\lambda_{1} & 2.846
\end{array}
$$

vertical socale $5(1) \mathrm{m} / \mathrm{l}) \mathrm{v}$.

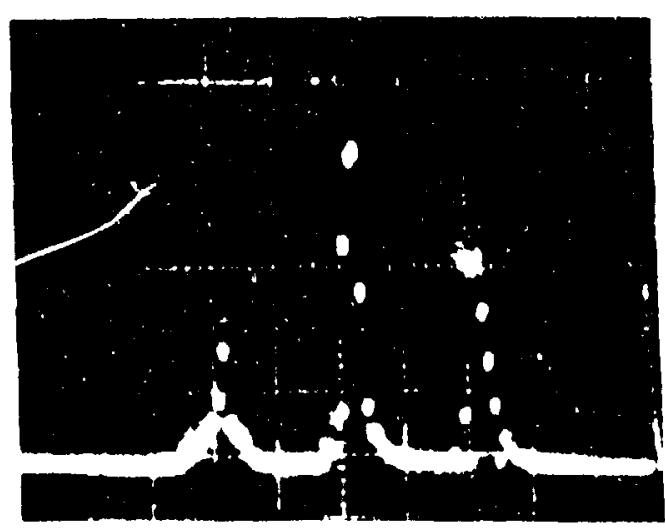

(b)

$$
\begin{aligned}
& \lambda_{1}=3.045 \mu \mathrm{m}, \\
& \lambda_{2}=3.94 \mu \mathrm{m}, \\
& \lambda_{3}=2.836 \mu \mathrm{m},
\end{aligned}
$$

vertical scale $=50 \mathrm{mV} /$ Div.

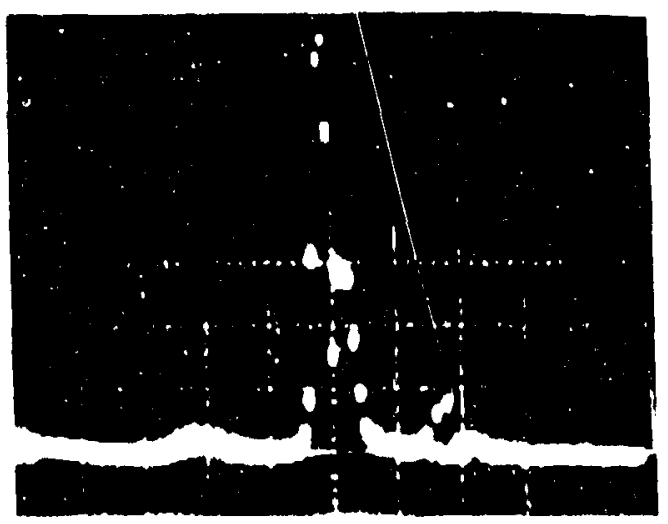

(d)

$\because \quad 30.4 \mathrm{MeV}$

$\lambda_{1} 3.06 \mathrm{hm}$,

$\lambda_{2} 2.0565 \mathrm{~s} \mu \mathrm{m}$

$\lambda$, $2.850,6 \mathrm{~mm}$.

vertical scale $\quad 50 \mathrm{mV} / \mathrm{l}$ )iv.

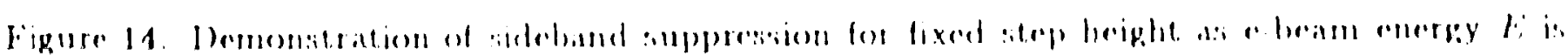
tunned from 39.9 M.V the 3!9.4 MeV. 